SCIPP-92/30

July, 1992

\title{
Spontaneous CP violation in Supersymmetric theories
}

\author{
Alex Pomarol \\ Santa Cruz Institute for Particle Physics \\ University of California, Santa Cruz, CA 95064
}

\begin{abstract}
We study the minimal version of the supersymmetric standard model with spontaneous $\mathrm{CP}$ breaking. In this model, the $\mathrm{KM}$ matrix is real and contributions to $\varepsilon$ arise from box diagrams involving squarks. We analyze the region of the parameter space which corresponds to values for $\varepsilon, \varepsilon^{\prime} / \varepsilon$ and the neutron electric dipole moment (NEDM) in agreement with the experimental data. We show that the $\mathrm{CP}$ violating phases must be of $\mathcal{O}\left(10^{-2}\right)$ and the NEDM lies near its present experimental limit.
\end{abstract}




\section{Introduction}

The origin of CP nonconservation is not yet fully understood. One major concern in recent times has been to understand why in the standard model (SM) the $\mathrm{CP}$ violating phase of QCD, $\bar{\theta}$, is so small (i.e., the strong CP problem). Several solutions to this puzzle have been proposed in the literature. The most appealing idea is the Peccei-Quinn (PQ) mechanism ${ }^{1}$.

In supersymmetric theories, however, even if one can arrange for $\bar{\theta} \simeq 0$, a new problem arises. It has been known for a long time that the supersymmetric extension of the SM contains a number of new sources of CP violation whose contribution to the neutron electric dipole moment (NEDM) is two or three orders of magnitude larger than the experimental limit if the phases that parametrize the $\mathrm{CP}$ violation, $\varphi$, are of $\mathcal{O}(1)^{2--7}$. Thus, a fine-tuning of parameters is necessary such that $\varphi \lesssim 10^{-2}-10^{-3}$. Since such CP violating phases arise from different sectors of the supersymmetric model, this multiple fine-tuning appears to be totally unnatural. In fact, it violates 't Hooft's naturalness condition which states that a parameter is only allowed to be very small if setting it to zero increases the symmetry of the theory ${ }^{8}$.

One simple and very attractive solution to this problem is to require that $\mathrm{CP}$ is spontaneously broken. In this case, CP invariance is imposed on the initial Lagrangian and it is broken by the ground state along with the gauge symmetry. One example in which such idea is implemented is the supersymmetric version ${ }^{9}$ of the Barr and Nelson models ${ }^{10}$. Models of this type require the existence of exotic superheavy fermions which mix with the standard light fermions. At low energy, such models are indistinguishable from the Kobayashi-Maskawa (KM) model. Another example is given in ref. [11] where CP is spontaneously broken at a high 
energy scale inducing complex scalar mass terms at low energy. In such a model, extra color singlet and color triplet fields are necessary.

In this paper we analyze the minimal version of the supersymmetric standard model with spontaneous $\mathrm{CP}$ violation $(\mathrm{SCPV})$. In such a model, $\mathrm{CP}$ violation derives from the phases of the vacuum expectation values (VEVs) of the Higgs bosons. The purpose of this work is to determine whether this model can explain the $\mathrm{CP}$ nonconservation observed in the $K-\bar{K}$ system while being consistent with the present bounds on the NEDM.

It has been claimed for a long time that supersymmetric models need the KM phase in order to explain the $\mathrm{CP}$ violating phenomena ${ }^{12}$. This statement is based on examining spontaneously broken $\mathrm{N}=1$ supergravity theories with a flat Kähler metric (i.e., all the scalar kinetic terms are canonical). In these theories

$$
\Delta m_{\tilde{q}}^{2} \equiv\left(m_{\tilde{q}_{1}}^{2}-m_{\tilde{q}_{2}}^{2}\right) \sim\left(m_{q_{1}}^{2}-m_{q_{2}}^{2}\right),
$$

where $\tilde{q}_{1}$ and $\tilde{q}_{2}$ are the scalar-partners (squarks) of the $q_{1}$ and $q_{2}$ quarks respectively. Since box diagrams involving superpartners (superbox) are suppressed by a factor $\left(\Delta m_{\tilde{q}}^{2} / m_{\tilde{q}}^{2}\right)^{2}$ due to the so-called super-GIM mechanism, their contributions to $\varepsilon$ are negligible for $\varphi \lesssim 10^{-2}$.

When more general $\mathrm{N}=1$ supergravity theories are considered, eq. (1) is no longer satisfied ${ }^{13}$ and superbox diagrams can be phenomenologically important. In fact, in such theories, the squark mass matrix is completely arbitrary, and as a result its diagonalization is independent of the diagonalization of the quark matrix. This implies that the unitary matrices, $V^{a}$, which characterize the Higgs or gauge fermionic-partners (higgsino or gaugino, $\tilde{\chi}_{a}$ ) interactions with quarks and squarks, 
i.e.,

$$
\mathcal{L}_{i n t} \propto V_{i j}^{a} \bar{q}_{i} \tilde{q}_{j}\left(1-\gamma_{5}\right) \tilde{\chi}_{a}+\text { h.c. }
$$

are arbitrary.

In this paper we will work within the context of such general $\mathrm{N}=1$ supergravity theories. Our results, however, can be easily generalized to a wide class of effective low-energy supersymmetric models. ${ }^{\# 1}$ Following ref. [15], we will assume approximately diagonal forms for the super-KM matrices, $V^{a}$, similar to the standard KM matrix:

$$
V^{a} \simeq\left(\begin{array}{ccc}
1 & \mathcal{O}\left(\sin \theta_{c}\right) & \mathcal{O}\left(10^{-2}\right) \\
\mathcal{O}\left(\sin \theta_{c}\right) & 1 & \mathcal{O}\left(\sin \theta_{c}\right) \\
\mathcal{O}\left(10^{-2}\right) & \mathcal{O}\left(\sin \theta_{c}\right) & 1
\end{array}\right)
$$

where $\theta_{c}$ is the Cabibbo angle. Even with this natural assumption, the contribution of the superbox diagrams to flavor changing neutral current (FCNC) processes is too large unless there is some mass degeneracy between squarks. Bounds on $\Delta m_{\tilde{q}}^{2} / m_{\tilde{q}}^{2}$ from FCNC processes were studied many years ago in refs. $[15,16]$. A recent analysis can be found in ref. [17]. Possible origins for such a degeneracy have been explored in ref. [18].

\section{The Higgs sector of the Model}

The minimal supersymmetric extension of the standard model (MSSM) requires two Higgs doublets. The VEVs of the two neutral scalars can be chosen real without loss of generality ${ }^{19}$ so that CP cannot be spontaneously broken. It has

\#1 It has been recently emphasized ${ }^{14}$ that the idea of supersymmetry at the weak scale should be tested without regard to the Planck-scale origin of any specific model. 
been recently claimed that SCPV can occur in the MSSM when radiative corrections to the Higgs potential are included ${ }^{20,21}$. This, however, requires ${ }^{21}$ a Higgs boson lighter than that permitted by the LEP Higgs search ${ }^{22}$. An extension of the MSSM Higgs sector is thus required if we want to have SCPV in supersymmetric theories.

Let us consider a model with two Higgs doublets $H_{1} \equiv\left(H_{1}^{0}, H_{1}^{-}\right)$and $H_{2} \equiv$ $\left(H_{2}^{+}, H_{2}^{0}\right)$ with hypercharges $Y=-1$ and $Y=1$ respectively and a complex singlet $N$. Such a Higgs sector has been extensively studied in the literature ${ }^{23,24}$ and provides an attractive solution to the $\mu$-problem. The most general renormalizable and gauge invariant superpotential for one quark generation is given by

$$
\begin{aligned}
W & =\frac{1}{3} \lambda_{1} N^{3}+\lambda_{2} H_{1} H_{2} N+\frac{1}{2} \mu_{N} N^{2}+\mu H_{1} H_{2} \\
& +h_{d} H_{1} \tilde{Q} \tilde{D}^{c}+h_{u} H_{2} \tilde{Q} \tilde{U}^{c}
\end{aligned}
$$

where $\tilde{Q}$ is the squark doublet, $\tilde{U}$ and $\tilde{D}$ are the squark singlets, and we have fixed the notation such that $H_{1} H_{2} \equiv H_{1}^{0} H_{2}^{0}-H_{1}^{-} H_{2}^{+}$. The scalar potential in the supersymmetric limit is given by

$$
V=\frac{1}{2}\left[\sum_{a}\left(\frac{1}{2} g A_{i}^{*} \sigma_{i j}^{a} A_{j}\right)^{2}+\left(\frac{1}{2} g^{\prime} Y_{i} A_{i}^{*} A_{i}\right)^{2}\right]+\left|\frac{\partial W}{\partial A_{i}}\right|^{2},
$$

where $A_{i}$ collectively denotes all scalar fields appearing in the theory. After spontaneous supergravity breaking, new terms are induced in the low-energy Higgs potential which softly break global supersymmetry (SUSY). These are given by ${ }^{13}$

$$
\begin{aligned}
V_{\text {soft }} & =m_{1}^{2}\left|H_{1}\right|^{2}+m_{2}^{2}\left|H_{2}\right|^{2}+m_{3}^{2}|N|^{2}+m_{12}^{2} H_{1} H_{2} \\
& +m_{N}^{2} N^{2}+A_{N} N H_{1} H_{2}+A_{N}^{\prime} N^{3}+\text { h.c. }
\end{aligned}
$$

$\mathrm{CP}$ invariance implies that all couplings and mass parameters are real. In order to have the desired pattern of gauge symmetry breaking, we will assume that only 
the neutral components of the Higgs bosons develop VEVs:

$$
\left\langle H_{1}^{0}\right\rangle=v_{1}, \quad\left\langle H_{2}^{0}\right\rangle=v_{2} e^{i \rho}, \quad\langle N\rangle=n e^{i \xi}
$$

For $\rho, \xi \neq n \pi(n \in \mathcal{Z}), \mathrm{CP}$ is broken along with the gauge symmetry. The phasedependent part of the Higgs boson potential can be written as

$$
\begin{aligned}
V(\rho, \xi) & =A \cos \xi+B \cos 2 \xi+C \cos 3 \xi+D \cos \rho \\
& +E \cos (\rho-2 \xi)+F \cos (\rho+\xi)
\end{aligned}
$$

where the new $A, B, C, D, E$ and $F$ quantities can be easily related to the original parameters. It can be shown that there exists a region in the parameter space where the minimum of the potential is at $\rho, \xi \neq n \pi .^{\# 2}$ From eq. (8), we see that when $\xi$ is small, $\rho$ must be close to 0 or $\pi$. This means that only one finetuning, $\xi \ll 1$, will be necessary in order that all $\mathrm{CP}$ violating effects are small; we will see that this is required by the NEDM bound. The smallness of $\xi$ does not violate 't Hooft's naturalness condition.

In our model, the $\mathrm{CP}$ violating processes will always involve neutral Higgs boson couplings. Prior to spontaneous gauge symmetry breaking, the neutral Higgs interactions with fermions are given by (following the notation of ref. [23])

$$
\begin{aligned}
\mathcal{L}_{\text {int }} & =-h_{u} H_{2}^{0} \bar{u}_{R} u_{L}-h_{d} H_{1}^{0} \bar{d}_{R} d_{L} \\
& -g\left(H_{1}^{0 *} \overline{\tilde{H}} P_{L} \tilde{W}+H_{2}^{0 *} \overline{\tilde{W}} P_{L} \tilde{H}\right)-\sqrt{\frac{1}{2}}\left(H_{1}^{0 *} \tilde{\tilde{H}}_{1}-H_{2}^{0 *} \tilde{\tilde{H}}_{2}\right) P_{L}\left(g \tilde{W}_{3}-g^{\prime} \tilde{B}\right) \\
& -\lambda_{2}\left(H_{1}^{0} \overline{\tilde{N}} P_{L} \tilde{H}_{2}+H_{2}^{0} \overline{\tilde{N}} P_{L} \tilde{H}_{1}+N \overline{\tilde{H}}_{1} P_{L} \tilde{H}_{2}-N \overline{\tilde{H}} P_{L} \tilde{H}\right) \\
& -2 \lambda_{1} N \overline{\tilde{N}} P_{L} \tilde{N}+\text { h.c. },
\end{aligned}
$$

where $P_{L}=\left(1-\gamma_{5}\right) / 2$, and the relevant neutral Higgs interactions with squarks

\#2 This is only true for the most general superpotential of eq. (4) or for theories beyond the minimal $\mathrm{N}=1$ supergravity ${ }^{25}$. 
are given by $\# 3$

$$
\begin{aligned}
\mathcal{L}_{i n t} & =h_{u}\left(\lambda_{2} H_{1}^{0 *} N^{*}+\mu H_{1}^{0 *}+m_{6} A_{u} H_{2}^{0}\right) \tilde{u}_{R}^{*} \tilde{u}_{L} \\
& +h_{d}\left(\lambda_{2} H_{2}^{0 *} N^{*}+\mu H_{2}^{0 *}+m_{6} A_{d} H_{1}^{0}\right) \tilde{d}_{R}^{*} \tilde{d}_{L}+\text { h.c. }
\end{aligned}
$$

When the neutral Higgs bosons develop VEVs, the interactions of eq. (9) and eq. (10) induce complex mass terms for the gauginos, higgsinos, quarks and squarks. ${ }^{\#}$ Since the phases $\rho$ and $\xi$ cannot be rotated away, CP is violated by the fermion and scalars propagators. The gauginos and higgsinos mix with each other, and the resulting mass eigenstates are called charginos $\left(\tilde{\chi}^{+}\right)$and neutralinos $\left(\tilde{\chi}^{0}\right)$. Notice that our model is similar to the supersymmetric model with explicit $\mathrm{CP}$ violation. However, there are some important differences. The KM matrix is now real ${ }^{26}$ as is the gluino mass $\left(m_{g}\right)$. Moreover, all $\mathrm{CP}$ violating phases, $\varphi$, can be written as a function of only the two phases $\rho$ and $\xi$, i.e., $\varphi=\varphi(\rho, \xi)$.

\section{Contributions to $\varepsilon, \varepsilon^{\prime}$ and the NEDM}

In this section we calculate the predictions of the model described in the previous section for the $\varepsilon$ and $\varepsilon^{\prime}$ parameters and the NEDM. Following the notation of ref. [27], we have

$$
\begin{aligned}
\varepsilon & =\frac{1}{\sqrt{2}} e^{i \pi / 4}\left(\frac{1}{2} t_{m}+t_{0}\right) \\
\varepsilon^{\prime} & =-\frac{1}{\sqrt{2}} i e^{i\left(\delta_{2}-\delta_{0}\right)} \frac{\operatorname{Re} A_{2}}{\operatorname{Re} A_{0}} t_{0}
\end{aligned}
$$

where $A_{i}$ are the weak-decay amplitudes of the neutral kaon to two pions of isospin

\footnotetext{
\#3 The coefficients of the soft-breaking terms $H_{i} \tilde{q} \tilde{q}$ are in principle arbitrary ${ }^{13}$. In agreement with standard theoretical prejudices ${ }^{14}$, we will assume that these coefficients are proportional to the Yukawa coupling $h_{q}$. Otherwise contributions to the NEDM will be too large. \#4 Neutral Higgs complex mass terms are also induced. For small $\varphi$, however, they do not give rise to any significant phenomenological implication.
} 
$i, \delta_{i}$ are the corresponding phases from strong interactions and

$$
t_{i}=\frac{\operatorname{Im} A_{i}}{\operatorname{Re} A_{i}}, \quad t_{m}=\frac{\operatorname{Im} M_{12}}{\operatorname{Re} M_{12}},
$$

where $M_{i j}$ is the neutral kaon mass matrix in the $K^{0}-\bar{K}^{0}$ basis. We have used the phase convention such that $t_{2}=0$. From the experimental values ${ }^{22,28}$

$$
\begin{aligned}
& |\varepsilon| \simeq 2.26 \cdot 10^{-3}, \\
& \varepsilon^{\prime} / \varepsilon \lesssim 1.45 \cdot 10^{-3}, \\
& \left|A_{2} / A_{0}\right| \simeq 1 / 22, \\
& \delta_{2}-\delta_{0} \simeq-53^{0},
\end{aligned}
$$

we have

$$
\begin{aligned}
t_{m} & \simeq 2 \sqrt{2}|\varepsilon| \simeq 6 \cdot 10^{-3}, \\
t_{0} & \simeq \sqrt{2}\left|\frac{A_{0}}{A_{2}}\right|\left|\varepsilon^{\prime}\right| \lesssim 10^{-4} .
\end{aligned}
$$

To begin with, let us consider the contribution to $t_{m}$. The only diagrams that considerably contribute to $\operatorname{Im} M_{12}$ are those involving phases in the propagators of the superpartners. In order to have a complex $\tilde{\chi}^{0}$ or $\tilde{\chi}^{+}$propagator, it is easy to see from eq. (9) that mixing between gauginos and higgsinos is required. As a result, the superbox diagrams must involve a quark-squark-higgsino coupling so that their contribution to $\operatorname{Im} M_{12}$ is suppressed by a factor $m_{q} / m_{W}$. If the phases arise from a squark propagator, then $\tilde{q}_{L}-\tilde{q}_{R}$ mixing is necessary [see eq. (10)]. In this case, superbox diagrams receive a suppression factor $m_{q} / m_{\tilde{q}}$. Thus, only superbox diagrams involving $\tilde{t}$ are nonnegligible. The largest of these contributions arise from the diagrams shown in fig. 1. In addition, there are two more diagrams like those of fig. 1 but with $\tilde{H}$ and $\tilde{W}$ interchanged in the $\tilde{H}-\tilde{W}$ fermion line, and 
contributing an opposite phase. Since these diagrams involve different super-KM matrix elements, there will be only a partial cancellation, which we denote by $S$. In order that the contribution of the diagrams of fig. 1 be large enough, we need a small mass for the lightest chargino and squark. In such a case, the contribution to $\operatorname{Re} M_{12}$ given by the diagram shown in fig. 2 is also large and a degeneracy between $\tilde{u}_{L}$ and $\tilde{d}_{L}$ is required in order to be consistent with the experimental value. ${ }^{~}$ From ref. [15], we have, for $m_{\tilde{\chi}^{+}} \sim m_{\tilde{q}} \sim 100 \mathrm{GeV}$,

$$
\frac{\Delta m_{\tilde{\tilde{q}}}^{2}}{m_{\tilde{q}}^{2}} \lesssim \mathcal{O}\left(\frac{1}{30}\right)
$$

When the bound (16) is saturated, the value of $t_{m}$ is given by the ratio between the diagrams of fig. 1 and fig. 2. A rough calculation gives

$$
t_{m} \simeq \frac{\frac{m_{t}}{\sqrt{2} m_{W} \sin \beta} V_{13} S \sin \varphi}{\Delta m_{\tilde{q}}^{2} / m_{\tilde{q}}^{2}},
$$

where $\tan \beta=v_{2} / v_{1}$ and $V_{13}$ is, according to eq. (3), of $\mathcal{O}\left(10^{-2}\right)$. We have assumed the maximal $\tilde{H}-\tilde{W}$ and $\tilde{t}_{L^{-}} \tilde{t}_{R}$ mixing. This is a natural assumption for $m_{\tilde{\chi}^{+}} \sim$ $m_{t} \sim 100 \mathrm{GeV}$. The fact that the diagrams of fig. 1 involve the $\bar{d} P_{R} \tilde{H}^{c} \tilde{t}_{R}$ coupling which is proportional to $\frac{m_{t}}{\sqrt{2} m_{W} \sin \beta}$ is crucial: the super-GIM mechanism does not apply and such diagrams receive only one power of the suppression factor $\Delta m_{\tilde{q}}^{2} / m_{\tilde{q}}^{2}$. For $\tan \beta \simeq 1, m_{t} \simeq 2 m_{W}$ and $S \simeq 1 / 2$, we have

$$
t_{m} \simeq 3 \cdot 10^{-1} \sin \varphi
$$

Since this is the maximal contribution to $t_{m}$, we have from eq. (15) the lower bound

$$
\varphi \gtrsim 2 \cdot 10^{-2}
$$

\#5 The contribution to $\operatorname{Re} M_{12}$ from superbox diagrams involving neutralinos and gluinos can be neglected if the masses of these particles are larger than $200 \mathrm{GeV}^{17}$. 
To estimate $t_{0}$, we assume that $A_{0}$ is dominated by the penguin diagrams ${ }^{29}$. The largest contribution to $\operatorname{Im} A_{0}$ arises from penguin diagrams involving charginos and top squarks (fig. 3). The chargino penguin diagrams also contribute to $\operatorname{Re} A_{0}$. The dominant contribution is shown in fig. 4 and leads to the effective lagrangian $\left(\text { for } m_{\tilde{\chi}^{+}} \sim m_{\tilde{q}}\right)^{30}$

$$
\mathcal{L}_{s p} \simeq \frac{\alpha_{s} \alpha_{W}}{24 m_{\tilde{q}}^{2}} \sin \theta_{c} \frac{\Delta m_{\tilde{q}}^{2}}{m_{\tilde{q}}^{2}} \mathbf{O}_{L R}+\text { h.c. }
$$

where $\mathbf{O}_{L R}=\left(\bar{s}_{L} \gamma_{\mu} T^{a} d_{L}\right)\left(\bar{q}_{R} \gamma^{\mu} T^{a} q_{R}\right)$, and $T^{a}$ is the hermitian $\mathrm{SU}(3)_{c}$ generator. Considering only the chargino contribution, the ratio $t_{0}$ is found to be of the same order of the ratio $t_{m}$ given in eq. (17). However, the dominant contribution to $\operatorname{Re} A_{0}$ arises from the standard penguin diagram:

$$
\mathcal{L}_{p} \simeq \frac{\alpha_{s} \alpha_{W}}{3 m_{W}^{2}} \sin \theta_{c} \ln \frac{m_{c}^{2}}{m_{K}^{2}} \mathbf{O}_{L R}+\text { h.c. }
$$

Therefore,

$$
t_{0} \simeq \frac{m_{W}^{2}}{16 m_{\tilde{q}}^{2}} \frac{\frac{m_{t}}{\sqrt{2} m_{W} \sin \beta} V_{13} S \sin \varphi}{\ln \left(m_{c}^{2} / m_{K}^{2}\right)}
$$

For the same values of the parameters considered in obtaining eq. (18), we have $t_{0} \sim 6 \cdot 10^{-6}$ in agreement with the experimental limit given in eq. (15). We must remark that the predictions for $t_{0}$ have large uncertainties ${ }^{31}$ and cannot be considered a precision test of the model.

Constraints from the NEDM, $d_{n}$, are more severe. The predictions of our model for $d_{n}$ can be estimated using previous calculations of the NEDM in supersymmetric models with explicit CP violation. Such calculations can be found in refs. [2-4]; more recent analyses are given in refs. [5-7]. The dominant contribution to $d_{n}$ arises from diagrams involving gluinos. Although the $\mathrm{CP}$ violating phase that appears 
in such diagrams is different from the phase that appears in fig. 1, both are of the same order (assuming no accidental cancellation). Different contributions to the NEDM arising from the induced quark electric dipole moment, quark chromoelectric dipole moment, Weinberg's three-gluon operator and one-photon-threegluon operator have been considered in ref. [6]. Using the experimental value ${ }^{32}$ $\left|d_{n}\right|<1.2 \cdot 10^{-25} \mathrm{e} \mathrm{cm}$, the tightest bound found in ref. [6] for $m_{g} \sim m_{\tilde{q}} \sim m_{Z}$ is $\varphi \lesssim 7.5 \cdot 10^{-3}$.

The next most important contribution to the NEDM comes from diagrams involving charginos (e.g., fig. 5). For $m_{\tilde{d}} \sim m_{\tilde{\chi}^{+}}$, this contribution is given by ${ }^{3}$

$$
d_{n} \simeq \frac{e g^{2}}{36 \sqrt{2} \pi^{2} m_{W} \cos \beta} \frac{m_{d}}{m_{\tilde{d}}} \sin \varphi
$$

which also gives rise to an upper bound for $\varphi$ of $\mathcal{O}\left(10^{-2}\right)$. The fact that these bounds are so close to that of eq. (19) suggests that a more rigorous calculation should be carried out in order to determine whether this model is ruled out. Notice, however, that we still have enough freedom in the parameter space to decrease the contribution to the NEDM without decreasing the contribution to $t_{m}$ coming from the diagrams of fig. 1. For example, constraints on $\varphi$ from gluino contributions to the NEDM can be made less severe by taking a larger gluino mass (if $m_{g} \gtrsim 300$ $\mathrm{GeV}$, the bound relaxes to $\varphi \lesssim 10^{-1}$ ). In the chargino case, contributions to the NEDM are smaller in the region of small $\tan \beta$ or small soft-supersymmetrybreaking gaugino mass term, $M<m_{W}^{\# 6}$ In addition, contributions to $t_{m}$ depend strongly on the super-KM matrices [see eq. (17)] which are in principle arbitrary.

\section{Conclusions}

\#6 Note that in the limit $M \rightarrow 0$ the phases in diagram shown in 5 can be rotated away giving a zero contribution to the NEDM. 
Even if $\bar{\theta}$ is small due to a PQ symmetry, a massless quark or some other possible mechanism, supersymmetric theories must still face the problem of having additional $\mathrm{CP}$ violating phases that induce a too large $d_{n}$.

In this paper we have proposed a supersymmetric model where CP is broken spontaneously. For this purpose, an additional scalar singlet is required. Phases in the VEVs of the neutral Higgs bosons are then responsible for all $\mathrm{CP}$ violating phenomena. They induce at low energy complex mass matrices for squarks, charginos and neutralinos. The main contribution to $\varepsilon$ arises from superbox diagrams (fig. 1). We showed that if $\Delta m_{\tilde{q}}^{2} / m_{\tilde{q}}^{2}$ saturates the bound derived from experimental limits from FCNC processes, such diagrams can explain the experimental value of $\varepsilon$. Contributions to $\varepsilon^{\prime} / \varepsilon$ and the NEDM are in agreement with the experimental bounds if the gluino mass is larger than about $200 \mathrm{GeV}$ and the $\mathrm{CP}$ violating phases are of $\mathcal{O}\left(10^{-2}\right)$. The smallness of these phases is natural in the sense of 't Hooft.

Deviations from the SM predictions are expected to be important in CP violating B decays. Such processes will be crucial for revealing the detailed structure of this model. An analysis of the impact of different classes of supersymmetric models on B decays can be found in ref. [33].

We must admit that our model suffers from the usual domain wall problems just like most models with SCPV. A possible solution which avoids such problems has been recently suggested in ref. [34].

Finally, it is interesting to note that the above analysis shows that $\mathrm{CP}$ violation can arise generically as a supersymmetric effect at low energy. In other words, the $\mathrm{KM}$ phase is not strictly necessary to explain the experimental observed $\mathrm{CP}$ violating phenomena. However, such a picture is consistent only for a small region 
of the parameter space of the supersymmetric model.

\section{Acknowledgements}

I would like to thank F. del Aguila, M. Dine, H. Haber and Y. Nir for helpful discussion and comments. I am also grateful to M. Dine and H. Haber for a critical reading of the manuscript. This work was supported by a fellowship of the MEC (Spain). 


\section{REFERENCES}

1. R.P. Peccei and H.R. Quinn, Phys. Rev. Lett. 38 (1977) 1440; Phys. Rev. D16 (1977) 1791.

2. J. Ellis, S. Ferrara and D.V. Nanopoulos, Phys. Lett. B114 (1982) 231;

W. Buchmüller and D. Wyler, Phys. Lett. B121 (1983) 321.

3. J. Polchinski and M. B. Wise, Phys. Lett. B125 (1983) 393.

4. F. del Aguila, M.B. Gavela, J.A. Grifols, and A. Méndez, Phys. Lett. B126 (1983) 71 ;

D.V. Nanopoulos and M. Srednicki, Phys. Lett. B128 (1983) 61;

A.I. Sanda, Phys. Rev. D32 (1985) 2992.

5. E. Braaten, C.S. Li and T.C. Yuan, Phys. Rev. Lett. 64 (1990) 1709;

J. Dai et al., Phys. Lett. B237 (1990) 216;

M. Dine and W. Fischler, Phys. Lett. B242 (1990) 239;

R. Arnowitt, J.L. Lopez and D.V. Nanopoulos, Phys. Rev. D42 (1990) 2423;

R. Arnowitt, M.J. Duff and K.S. Stelle, Phys. Rev. D43 (1991) 3085.

6. A. De Rújula, M.B. Gavela, O. Pène and F.J. Vegas, Phys. Lett. B245 (1990) 640.

7. Y. Kizukuri and N. Oshimo, Phys. Rev. D45 (1992) 1806.

8. G. 't Hooft, 1979 Cargese Summer Institute Lectures.

9. S.M. Barr and A. Masiero, Phys. Rev. D38 (1988) 366.

10. A. Nelson, Phys. Lett. B136 (1984) 387; Phys. Lett. B143 (1984) 65;

S.M. Barr, Phys. Rev. Lett. 53 (1984) 329; Phys. Rev. D30 (1984) 1805.

11. A. Dannenberg, L. Hall and L. Randall, Nucl. Phys. B271 (1986) 574. 
12. F. del Aguila et al., Phys. Lett. B129 (1983) 77;

J.M. Frère and M.B. Gavela, Phys. Lett. B132 (1983) 107;

J.M. Gérard, W. Grimus, A. Raychaudhuri and G. Zoupanos, Phys. Lett.

B140 (1984) 349;

P. Langacker and B. Sathiapalan, Phys. Lett. B144 (1984) 401.

13. S.K. Soni and H.A. Weldon, Phys. Lett. B216 (1983) 215.

14. L.J. Hall and L. Randall, Phys. Rev. Lett. 65 (1990) 2939.

15. J. Ellis and D.V. Nanopoulos, Phys. Lett. B110 (1982) 44.

16. M.J. Duncan and J. Trampetic, Phys. Lett. B134 (1984) 439.

17. F. Gabbiani and A. Masiero, Nucl. Phys. B322 (1989) 235.

18. M. Dine, A. Kagan and S. Samuel, Phys. Lett. B243 (1990) 250.

19. See, for example J.F. Gunion, G.L. Kane, H.E. Haber and S. Dawson, The Higgs Hunter's Guide (Addison-Wesley Publishing Company, Reading, MA, 1990) for a comprehensive review and a guide to the literature.

20. N. Maekawa, Preprint KUNS 1124 (1992).

21. A. Pomarol, Preprint SCIPP-92/19 (1992).

22. Particle Data Group, Phys. Rev. D45 (1992) S1.

23. J.F. Gunion and H.E. Haber, Nucl. Phys. B272 (1986) 1.

24. J. Ellis et al., Phys. Rev. D39 (1989) 844;

M. Drees, Int. J. Mod. Phys. A4 (1989) 3635;

P. Binétruy and C.A. Savoy, Phys. Lett. B277 (1992) 453.

25. J.C. Romão, Phys. Lett. B173 (1986) 309.

26. G.C. Branco, Phys. Rev. Lett. 44 (1980) 504.

27. L.L. Chau, Phys. Rep. 95 (1983) 1. 
28. J.M. Gérard, a talk given in the 15th Int. Symp. on Lepton-Photon Interactions at High Energies (Geneva, 1991).

29. M.A. Shifman, A.I. Vainshtein and V.I. Zakharov, Nucl. Phys. B120 (1977) 316 ;

F.J. Gilman and M.B. Wise, Phys. Lett. B83 (1979) 83.

30. P. Langacker and B. Sathiapalan, Phys. Lett. B144 (1984) 395.

31. G. Buchalla, A.J. Buras and M.K. Harlander, Nucl. Phys. B337 (1990) 313.

32. I.S. Altarev et al., JETP Lett. 44 (1986) 460;

K.F. Smith et al., Phys. Lett. B234 (1990) 191.

33. I.I. Bigi and F. Gabbiani, Nucl. Phys. B352 (1991) 309.

34. L.M. Krauss and S.J. Rey, Preprint NSF-ITP-92-03 (1992). 


\section{FIGURE CAPTIONS}

1) Dominant one-loop contribution to $\operatorname{Im} M_{12}$. We denote by $\bullet$ a $\tilde{H}-\tilde{W}$ or $\tilde{t}_{L}-\tilde{t}_{R}$ mixing.

2) Dominant one-loop contribution to $\operatorname{Re} M_{12}$.

3) Dominant one-loop contribution to $\operatorname{Im} A_{0}$. We denote by $\bullet$ a $\tilde{H}-\tilde{W}$ or $\tilde{t}_{L}-\tilde{t}_{R}$ mixing.

4) Chargino one-loop contribution to $\operatorname{Re} A_{0}$.

5) One-loop chargino diagram contributing to the NEDM. We denote by a cross a mass insertion in the fermion line. 